Cahiers de philosophie de l'université de

CAHIERS DE PHILOSOPHIE
DE LUNIVERSITE DE CAEN

Caen

$52 \mid 2015$

Le « je » empirique des philosophes

\title{
Ego et René Descartes
}

\section{Anne-Marie Boudot}

\section{OpenEdition}

Journals

Édition électronique

URL : https://journals.openedition.org/cpuc/547

DOI : 10.4000/cpuc.547

ISSN : 2677-6529

Éditeur

Presses universitaires de Caen

Édition imprimée

Date de publication : 31 décembre 2015

Pagination : $93-112$

ISBN : 978-2-84133-746-0

ISSN : 1282-6545

Référence électronique

Anne-Marie Boudot, "Ego et René Descartes », Cahiers de philosophie de l'université de Caen [En ligne], 52 | 2015, mis en ligne le 13 juin 2018, consulté le 01 mars 2023. URL : http:// journals.openedition.org/cpuc/547; DOI : https://doi.org/10.4000/cpuc.547

Creative Commons - Attribution - Pas d'Utilisation Commerciale 4.0 International - CC BY-NC 4.0 https://creativecommons.org/licenses/by-nc/4.0/ 


\title{
Ego et René Descartes
}

\begin{abstract}
Comment Descartes pourrait-il être absent d'un recueil sur le «je» des philosophes? Ses deux œuvres majeures - le Discours de la Méthode et les Méditations - sont écrites de bout en bout à la première personne du singulier, chose peu fréquente en philosophie: ne vise-t-elle pas l'universel? L'ouvrage de 1637 contient cette "Histoire de [son] esprit» promise de longue date à ses amis, puisque Guez de Balzac la lui réclame dès 1628 . Quant aux Méditations, comme déjà la $\mathrm{IV}^{\mathrm{e}}$ partie du Discours, chacun sait qu'elles découvrent l'Ego comme l'unique connaissance qui résiste au doute et le "point archimédique» qui permet la reconstruction du savoir.

C'est cette double présence du «Je» qui doit nous interroger. Le «Je» qui a fait ses études à La Flèche, études dont le Discours détaille le cursus; qui, parvenu à l'âge d'homme, a résolu de voyager pour lire dans «le grand livre du monde»; qui s'est trouvé un jour "dans un poêle» en Allemagne, c'est incontestablement Descartes et nul autre. Il n'en va pas de même du «Je» du «Je pense donc je suis» ou de l'Ego du «Ego sum, Ego existo». Pour y parvenir, il a fallu se dépouiller du corps et de toutes les particularités qui font que Pierre est Pierre et que René est René. Et il appartient à chacun de refaire le chemin qui, traversant la nuit du doute, découvre la vérité irrécusable de l'existence de sa pensée: «Cette proposition Je suis, j'existe est nécessairement vraie, toutes les fois que je la prononce, ou que je la conçois en mon esprit ${ }^{1}$.

Par là, Descartes ouvre une ère nouvelle dans l'histoire de la philosophie occidentale, celle qu'on nommera plus tard la philosophie du Sujet (même si ce terme est absent du corpus cartésien en son acception moderne) et dont Kant et Husserl devaient être les plus illustres représentants. On peut cependant se demander si en raison même de l'importance de la tradition qu'il inaugure, cette postérité ne risque pas de fausser notre lecture de Descartes. Parce qu'il n'introduit pas, comme Kant, la distinction du Sujet transcendantal et du moi empirique, qu'il n'aurait pas distingué l'Ego du moi
\end{abstract}

1. Première Méditation, C. Adam, P. Tannery (éd.), Paris, Vrin, 1996 (= AT), t. IX, p. 19; texte latin: t. VII, p. 25. 
partie du monde, il aurait en définitive perdu le bénéfice de sa découverte ${ }^{2}$. L'Ego de Descartes serait, en définitive, un Sujet transcendantal manqué.

C'est sans doute parce qu'on a prêté une attention exclusive au côté pré-kantien ou pré-phénoménologique qu'on a rarement pris pour thème explicite ces deux emplois du «Je»: «Je», moi René Descartes et «Je» sujet universel. Sans doute y a-t-on vu au mieux un simple procédé stylistique, au pire le signe de la confusion entre le Sujet et le moi empirique. Mon dessein n'est pas de me livrer ici à un commentaire de plus du Cogito cartésien ${ }^{3}$; mais de tenter de mettre en lumière les rapports qui existent entre l'homme Descartes - en son temps et avec sa situation singulière - et sa philosophie, et plus particulièrement sa conception du Je et de l'Homme. Peut-être découvrirons-nous que ces rapports sont multiples et complexes. Complexité qui n'est pas confusion.

Ainsi, mon dessein n'est pas d'enseigner ici la méthode que chacun doit suivre pour bien conduire sa raison, mais seulement de faire voir en quelle sorte j'ai tâché de conduire la mienne ${ }^{4}$.

On sait comment Kant énonce le problème que doit résoudre la Critique de la raison pure:

Comment une mathématique pure est-elle possible? Comment une Physique pure est-elle possible? À propos de ces sciences, puisqu'elles sont effectivement données, on peut bien se demander, assurément, comment elles sont possibles; car qu'il leur faille être possibles, c'est démontré par leur réalité effectives.

Cette question trouvera sa solution avec la théorie du Sujet transcendantal. Un tel questionnement n'est pas celui de Descartes et ne peut pas l'être. Si paradoxale que soit cette formulation, on pourrait dire que la science n'existe pas, et qu'il sait très bien comment elle est possible. "La" physique n'existe pas. Certes, Descartes n'est pas le premier savant des temps modernes; avant lui, il y a eu Copernic, Kepler et Galilée, pour ne citer que les plus grands. L'idée d'une mathématisation du «sublunaire» existe avant lui. Mais c'est une illusion rétrospective qui nous fait croire que «la» méthode scientifique fut «une révélation pour tous les physiciens».

2. E. Husserl, Méditations cartésiennes [1929], trad. G. Peiffer et E. Levinas, Paris, Vrin, 1980, p. 20: «Comment Descartes a manqué l'orientation transcendantale».

3. C'est par commodité que j'utilise cette formulation traditionnelle. Bien entendu, l'énoncé décisif est celui des Méditations: Ego sum, Ego existo (AT, VII, p. 27).

4. Discours de la méthode, AT, VI, p. 4.

5. E. Kant, Critique de la raison pure, Introduction et trad. A. Renaut, Paris, Aubier, 1997, p. 107. 
La naïveté propre aux Lumières pense que seuls l'ignorance, le préjugé ou l'obscurantisme peuvent s'opposer à la science nouvelle. En fait, au moment où Descartes entame ses premières recherches, il n'existe pas de paradigme au sens de Kuhn, c'est-à-dire d'accord de la communauté des savants sur ce que doivent être une hypothèse, un énoncé, une vérification, pour être «scientifiques». Pour s'en convaincre (et sans même mentionner l'étrange mélange d'astronomie et d'astrologie chez Kepler), il suffit de songer aux controverses sur le vide entre Pascal et Descartes (qui, en l'occurrence, n'est pas du «bon» côté): les rôles respectifs de l'analyse du concept de vide et de l'expérience ne sont pas les mêmes; non que Descartes rejette l'expérimentation, comme on le croit souvent - il aurait même suggéré l'expérience du Puy-de-Dôme - mais les manières d'interpréter les faits sont incompatibles. D'où un dialogue de sourds, inconcevable lorsqu'un paradigme est établi ${ }^{6}$.

Si tel est le moment dans l'histoire (la préhistoire?) des sciences, il importe de ne pas oublier que Descartes est lui-même un homme de science. C'est en cela que la méthode qu'il propose dans le Discours et met en œuvre dans ses travaux scientifiques est la sienne. Non la méthode reçue dans la communauté scientifique, et qu'il se bornerait à appliquer à de nouveaux phénomènes, mais la méthode qu'il invente et applique. On sait que le Discours de la méthode est suivi de trois «Essais» de cette méthode. "Essais", donc mise à l'épreuve. Sans la méthode, la Dioptrique, les Météores et la Géométrie n'existeraient pas; sans eux, rien ne prouverait (ou n'éprouverait?) la valeur de cette méthode. Est-ce à dire que cette mise à l'épreuve suffit? Dans l'article 205 de la IV $\mathrm{IV}^{\mathrm{e}}$ partie des Principes, Descartes utilisera l'image du cryptogramme: si, ayant affaire à un message codé, je fais une hypothèse sur la nature de ce code, plus long sera le texte auquel je parviendrais à donner un sens intelligible, plus grande sera la probabilité que j'ai bien trouvé le bon principe de lecture. Mais ce n'est là une «certitude morale». Il n'est pas question de s'en contenter.

Revenons aux commencements de la physique moderne. Comme JeanLuc Marion l'a montré dans Sur la théologie blanche de Descartes ${ }^{7}$, Kepler et Galilée, les «pères fondateurs » de cette physique, ont d'emblée pensé la mathématisation du monde comme la mise au jour de sa nature mathématique. Dieu a créé l'univers selon la loi du nombre. Ainsi, Galilée écrit:

La philosophie est écrite dans ce vaste livre qui se tient constamment ouvert devant nos yeux (je veux dire l'Univers), mais on ne peut l'entendre, si l'on

6. À la fin du siècle, le paradigme n'est pas vraiment constitué. Voir la réaction de Leibniz devant l'attraction newtonienne, perçue comme un retour aux qualités occultes.

7. J.-L. Marion, Sur la théologie blanche de Descartes, Paris, PUF, 1981. 
n'apprend d'abord la langue, et ne connaît les caractères dans lesquels il est écrit. Car il est écrit en langue mathématique, et les caractères en sont les triangles, les cercles et les autres figures géométriques, faute desquels il est humainement impossible d'en comprendre une seule parole ${ }^{8}$.

La vérité scientifique est donc interprétée comme mise au jour de l'essence même de l'Univers.

Qu'en est-il pour Descartes? Si nous prêtons attention à la première des Regulae ad directionem ingenii, nous avons l'impression que sa position est bien différente, et fait l'économie de toute référence à la «réalité» des choses. Alors que pour la tradition aristotélicienne l'hétérogénéité des sciences était fondée sur l'hétérogénéité des régions de l'Univers, Descartes en affirme l'unité; mais non pas, comme Galilée, en affirmant que le monde est en son entier soumis à la loi du nombre, mais en se fondant sur ce qu'il appelle, sans grand souci de rigueur conceptuelle, ingenium, intellectus ou mens. C'est parce que notre esprit est un et toujours le même que la science est une, quels que soient les objets auxquels elle s'applique. Aussi certains commentateurs ont-ils pu parler de "révolution copernicienne ${ }^{9}$.

Cette centration sur le sujet connaissant semble du reste impliquée par la place centrale donnée à la méthode, ainsi que par le modèle mathématique. Qu'est-ce en effet qu'une méthode, sinon l'indication de règles explicites pour construire un objet, qu'il s'agisse des règles de l'addition ou de la multiplication ou d'un dispositif expérimental? Ces règles sont applicables par tous, et c'est précisément ce qui différencie la connaissance scientifique de l'opinion, ou encore de l'inspiration du poète ou du devin, qui peuvent bien se dire "vraies", mais qui doivent être crues sur paroles. La connaissance scientifique paraît ainsi substituer l'intersubjectivité à l'adéquation à la «chose». En fait, Descartes a tacitement supposé que la connaissance que sa méthode élabore est «vraie», au seul sens qu'il tient pour recevable - et connu de tous - à savoir conforme à ce qui est ${ }^{10}$. S'il prend par la suite une conscience explicite de ce que Galilée admettait sans examen, c'est parce que sa propre contribution constitue un pas supplémentaire dans

8. Cité par J.-L. Marion, Sur la théologie blanche de Descartes, p. 210. Voir aussi Tycho-Brahé: «Il n'y a pas de doute que dans l'Univers tout est divinement agencé et ordonné selon une harmonie et une proportion déterminées, et peut donc être valablement compris tant par les nombres que par les figures, comme l'ont autrefois pressenti les Pythagoriciens et les Platoniciens» (cité par G. Simon, Kepler, astronome, astrologue, Paris, Gallimard, 1979, p. 237).

9. Par exemple N. Grimaldi, L'expérience de la pensée dans la philosophie de Descartes, Paris, Vrin, 1978, chap. 3: "La révolution copernicienne».

10. Lettre à Mersenne du 16 octobre 1639, AT, II, p. 56. Dans les Principes, l'article 205, qui parle de la "certitude morale», est suivi de l'article 206: «Et même qu'on en a une certitude plus que morale» (AT, VIII, p. 328 et IX, p. 234). 
la mathématisation de la physique. Galilée avait franchi un premier pas en traitant de manière semblable mouvements astronomiques, comme le mouvement des planètes, et mouvements terrestres, comme le roulement d'une bille sur un plan incliné; ce faisant, il avait aboli la distinction entre le supra- et le sublunaire. Certes, il avait affirmé le principe de la mathématisation de tous les phénomènes; mais, de fait, il n'avait guère étudié que des mouvements. Descartes (même s'il n'est pas le seul) joue en revanche un rôle décisif dans la mathématisation de ce qui semble par nature rebelle à la loi du nombre: la qualité.

Considérons la très remarquable Règle XII des Regulae. Elle propose un «code» (l'expression n'est pas de Descartes, mais elle est commode), qui consiste à désigner les diverses couleurs par des figures géométriques. Cette codification est permise et avantageuse, car

quel inconvénient y aurait-il, pour ne pas admettre inutilement un être nouveau, [...] à ne rien nier sans doute de ce qu'il a plu à d'autres de penser sur la couleur, mais à ne considérer en celles-ci, indépendamment de toute autre chose, que ce qui est figuré, à concevoir la différence qui est entre le blanc, le bleu, le rouge, etc. comme celle qui existe [entre différentes figures] ${ }^{11}$.

Ceci est tout à fait conforme à la théorie du signe, dont Descartes voit l'arbitraire $^{12}$ : la couleur rouge ne ressemble pas plus au mot « rouge» qu'à un carré ou à un rectangle; et comme le relève la suite du texte, ce codage est toujours possible, puisque les figures géométriques sont en nombre infini, et qu'il est donc loisible de désigner des qualités différentes par des figures différentes. Certes, mais à quoi bon? On perçoit l'embarras de Descartes, réduit à avancer un piètre argument: que la couleur soit ce qu'on voudra, elle est toujours étendue. Piètre argument, puisqu'on pourrait aussi bien utiliser un tel codage pour désigner non le blanc, le bleu et le rouge, mais des passions comme l'amour, la haine ou la colère. Sa conviction est en réalité manifeste: malgré la prudence du «que la couleur soit ce qu'on voudra", il est convaincu que l'étendue est l'essence de la couleur; que dans le monde extérieur, comme aussi bien dans notre œil et dans notre cerveau, il n'y a que matière diversement figurée et mue, la qualité n'étant que dans notre âme. Le « codage», loin d'être arbitraire, comme le sont les signes de la langue, est donc homogène à ce qui est codé. Mais cela, rien

11. AT, X, p. 413: «Quid igitur sequetur incommodi, si, caventes ne aliquod novum ens inutiliter admittamus [...], non negemus quidem de colore quidquid aliis placuerit, sed tantum abstrahamus ab omni alio, quam quod habeat figurae naturam, et concipiamus diversitatem, quae est inter album, coeruleum, rubrum, etc. "; j'utilise la traduction de Le Roy, Paris, Boivin, 1932.

12. Traité du Monde, ou Traité de la lumière, AT, XI, p. 3. 
dans les Regulae ne permet de l'affirmer. Ce n'est pas parce que je décide d'un code ou d'une méthode que je peux assurer qu'elle permet de mettre au jour l'essence des choses.

On sait que les Regulae sont demeurées inachevées et que, bien qu'il en ait conservé le manuscrit, Descartes n'en fait jamais mention. A-t-il perçu qu'il s'engageait dans une impasse? Quoi qu'il en soit, avec les célèbres lettres à Mersenne du printemps 1630 sur la création des vérités éternelles, c'est une pensée bien différente que nous découvrons: le point de départ n'est plus la méthode, mais la Métaphysique.

J'estime que ceux à qui Dieu a donné l'usage de [la] raison, sont obligés de l'employer principalement à le connaître et à se connaître eux-mêmes. C'est par là que j'ai tâché de commencer mes études, et je vous dirai que je n'eusse su trouver les fondements de la Physique, si je ne les eusse cherchés par cette voie ${ }^{13}$.

Quelle était cette première version de la Métaphysique? Le Cogito y figurait-il déjà ? En quoi consistaient ces démonstrations des vérités métaphysiques "plus évidentes que les démonstrations de Géométrie»? Nous l'ignorons. Quoi qu'il en soit, un certain nombre de conclusions se dégagent de cette rapide étude. En premier lieu, apparait ici le concept de «fondement», que nous retrouverons constamment dans le cartésianisme ultérieur, qui désigne non seulement ce qui est premier (comme l'était la méthode dans les Regulae), mais aussi le sol sur lequel repose l'édifice du savoir, sans lequel ce savoir ne pourrait prétendre à la vérité; et ce fondement ne peut être que la Métaphysique. En second lieu, c'est $s a$ pratique de la physique qui le conduit à prendre conscience de la nécessité d'un tel fondement: c'est justement parce qu'il a conscience de fonder la science, au sens où Henri IV a fondé le Collège de la Flèche, qu'il ne peut la fonder au sens de trouver le sol inébranlable que chercheront les Méditations. En troisième lieu, ce fondement est trouvé non dans le sujet (en quelque sens qu'on l'entende), mais en Dieu. Enfin, les lettres de 1630 qui affirment, pour la première fois, la nécessité d'un fondement métaphysique de la physique affirment avec force la différence infinie entre l'homme et Dieu. Dieu crée les vérités, alors que la passivité de l'entendement humain sera une thèse constante de Descartes. Nous pouvons connaître l'Univers parce que Dieu est le créateur et du monde et de nos esprits, non parce que nous participons à l'esprit divin, comme le supposait Galilée ${ }^{14}$. Nous voici loin du Sujet transcendantal.

13. Lettre à Mersenne du 15 avril 1630, AT, I, p. 145.

14. Sur ce point, voir J.-L. Marion, Sur la théologie blanche de Descartes, p. 210 sq. 
En quoi consiste cette Métaphysique qui fournit à la «philosophie naturelle» ses principes? Et quelle relation existe entre l'homme Descartes et l'Ego universel, sinon impersonnel ? C'est ce qu'il faut examiner à présent.

Tous ceux à qui Dieu a donné l'usage de cette raison sont obligés de l'employer principalement pour tâcher à le connaître et à se connaître eux-mêmes ${ }^{15}$.

Dans la lettre à Mersenne du 11 novembre 1640, Descartes s'exprime en ces termes:

J'ai envoyé hier ma Métaphysique [...]. Je n'y ai point mis de titre, mais il me semble que le plus propre sera de mettre Renati Descartes Meditationes de prima Philosophia; car je ne traite point en particulier de Dieu et de l'Ame, mais en général de toutes les premières choses qu'on peut connaître en philosophant ${ }^{16}$.

Ce texte contient une définition implicite de la Métaphysique: c'est la connaissance, par la raison, des êtres immatériels. Effectivement, la «philosophie première» a un contenu plus étendu, puisqu'elle comprend aussi la connaissance de l'essence des corps ( $\mathrm{V}^{\mathrm{e}}$ Méditation) et leur existence $\left(\mathrm{VI}^{\mathrm{e}}\right)$. C'est sans doute pour cela que les Principes de la Philosophie renverront la question de l'existence des choses matérielles dans la $\mathrm{II}^{\mathrm{e}}$ partie. Il demeure que Descartes lui-même parle ordinairement de son ouvrage comme de «[sa] Métaphysique», ainsi que nous le voyons dans le texte cité à l'instant. Au reste, le choix de l'une ou l'autre dénomination semble de peu d'importance, puisque, de fait, du Discours de la Méthode aux Principes en passant par les Méditations, il est bien certain qu'il n'existe pour Descartes d'autre chemin que celui qui, partant du doute radical, établit l'existence de l'âme, puis celle de Dieu.

Pourtant, ce n'est pas la même chose que de définir un savoir par sa fonction - assurer aux sciences leur fondement - ou par son objet, l'immatériel; de faire de la connaissance de soi-même et de Dieu un simple moyen au service des sciences - les racines de l'arbre qu'on cultive pour ses fruits ou d'en faire l'objet privilégié de son étude. Qu'en est-il exactement? Pour jeter quelque lumière sur cette question, il est bon de s'attarder un instant sur l'autre élément du titre: méditations. Sans doute n'y a-t-on pas porté une attention suffisante, ni perçu ce qu'il a de surprenant ${ }^{17}$. Qu'est-ce que

15. Lettre à Mersenne du 15 avril 1630, AT, I, p. 144.

16. AT, III, p. 235.

17. Comme D. Kambouchner le relève dans son commentaire: Les Méditations métaphysiques de Descartes, Paris, PUF, 2005, t. I, p. 137 sq. Son étude apporte de précieux éléments sur ce point. 
méditer? La méditation appartient d'abord à la sphère de la spiritualité, et plus particulièrement de la spiritualité à la spiritualité religieuse. Comme l'écrit D. Kambouchner:

Dans toute la tradition [...] qui remonte à Augustin, la méditation constitue l'exercice complexe par lequel une âme en quête de lumière et de régénération se prépare à recevoir l'illumination divine et à connaître l'union à Dieu dans l'amour. [Elle] occupe le milieu entre la pensée (cogitatio) et l'état ultime de contemplation (contemplatio) ${ }^{18}$.

La pratique de la méditation avait connu un renouveau considérable dans les premières années du XVII ${ }^{\mathrm{e}}$ siècle. Descartes, élevé par les Jésuites, en relation avec des Oratoriens, ne peut pas avoir ignoré ces pratiques. Il sait donc ce que «méditer» signifie.

Même séparée de son contexte religieux, la notion de méditation contient, semble-t-il, deux idées sans lesquelles elle n'a pas de sens. En premier lieu, l'idée de l'approfondissement, de différents niveaux de sens, idée peut-être issue de la distinction des différents sens de l'Écriture qui a commandé toute l'exégèse chrétienne. Plutôt qu'à l'alternative du vrai et du faux, c'est à celle du superficiel et du profond que nous avons ici affaire. Rien n'indique du reste que cette recherche ait un terme, et qu'on puisse parler ici de compréhension totale ou ultime. En deuxième lieu, la méditation implique une transformation intérieure du sujet méditant. Il ne s'agit pas seulement pour l'entendement d'être éclairé, mais pour l'âme entière d'être transformée. C'est pourquoi on peut voir dans les Confessions de saint Augustin la première des méditations de l'ère chrétienne.

Il est facile de mettre en lumière tous les passages qui, dans l'ouvrage de 1641, justifient son titre. Ainsi, le début de la $\mathrm{II}^{\mathrm{e}}$ Méditation, où le doute (qui, avec l'hypothèse du Malin Génie, semblait presque jeu de l'esprit) est vécu à présent sur le mode de l'angoisse:

La Méditation que je fis hier m'a rempli l'esprit de tant de doutes, qu'il n'est plus désormais en ma puissance de les oublier. Et cependant je ne vois pas de quelle façon je les pourrais résoudre; et comme si tout à coup j'étais tombé dans une eau très profonde, je suis tellement surpris, que je ne puis ni assurer mes pieds dans le fond, ni nager pour me soutenir au-dessus ${ }^{19}$.

Nous sommes loin du scepticisme souriant de Montaigne ou de l'incrédulité des libertins. Le début de la III ${ }^{\mathrm{e}}$ Méditation n'est pas sans évoquer l'effort du Chrétien pour se détacher du monde:

18. D. Kambouchner, Les Méditations métaphysiques de Descartes, p. 140.

19. AT, IX, p. 18, latin, AT, VII, p. 23-24. 
Je fermerai maintenant les yeux, je boucherai mes oreilles, je détournerai tous mes sens, j'effacerai même de ma pensée toutes les images des choses corporelles, ou du moins, parce qu'à peine cela se peut-il faire, je les réputerai comme vaines et comme fausses, et ainsi m'entretenant seulement moi-même, et considérant mon intérieur, je tâcherai de me rendre peu à peu plus connu, et plus familier à moi-même ${ }^{20}$.

Mais c'est, bien sûr, l'élévation finale de la III ${ }^{\mathrm{e}}$ Méditation qui correspond à ce qu'on entend par «méditation »; au terme d'une démarche qui fut celle de la raison, une pause, où l'âme entière s'élève et où la démonstration laisse la place à l'admiration et à la béatitude:

Mais auparavant que j'examine cela plus soigneusement, et que je passe à la considération des autres vérités que l'on peut recueillir, il me semble très à propos de m'arrêter quelque temps à la contemplation de ce Dieu tout parfait, de peser tout à loisir ces merveilleux attributs, de considérer, d'admirer, et d'adorer l'incomparable beauté de cette immense lumière, au moins autant que la force de mon esprit, qui en demeure en quelque sorte ébloui, me le pourra permettre.

Car comme la foi nous apprend que la Souveraine félicité de l'autre vie, ne consiste que dans cette contemplation de la Majesté divine: Ainsi expérimentons-nous dès maintenant, qu'une semblable Méditation quoique incomparablement moins parfaite, nous fait jouir du plus grand contentement que nous soyons capables de ressentir en cette vie ${ }^{21}$.

Il ne suffit pas, cependant, de relever les passages du texte cartésien qui illustrent l'idée de méditation. Il faut prendre conscience d'une difficulté: le discours méditatif s'oppose radicalement au discours démonstratif qui prend les mathématiques pour modèle; et, plus précisément, à la démonstration telle que Descartes lui-même la théorise.

Dès les Regulae, Descartes a mis au jour la nature de la démonstration mathématique, et jamais il ne reviendra sur ce point. La chaîne démonstrative prend son départ dans des propositions saisies par intuition, et c'est par intuition également que sont «vus» les enchaînements; entre l'intuition et la déduction, il n'existe de différence qu'eu égard aux limites de notre attention: je ne peux saisir uno intuitu une longue suite de propositions. Mais, de même que pour m'assurer de la continuité d'une chaîne, je dois voir que chaque anneau est solidement joint au précédent et au suivant, pour m'assurer de la valeur d'une démonstration, je dois «voir» la liaison nécessaire de chaque proposition avec celle dont elle dérive et avec celle

20. AT, IX, p. 27, latin, AT, VII, p. 34 .

21. AT, IX, p. 41-42, latin, AT, VII, p. 52. 
qu'on en tire. Bien que Descartes emprunte le terme d'intuitus à la sphère de la vision, il prend bien soin de marquer la différence:

Par intuition j'entends, non pas le témoignage changeant des sens ou le jugement trompeur d'une imagination mensongère, mais la conception d'un esprit pur et attentif, conception si facile et si distincte qu'aucun doute ne reste sur ce que nous comprenons; ou, ce qui est la même chose, la conception ferme d'un esprit pur et attentif, qui naît de la seule lumière de la raison et qui, étant plus simple, est par suite plus sûre que la déduction même ${ }^{22}$.

Le terme essentiel est ici celui de simple. La vue par les sens n'est pas seulement «trompeuse», elle est par nature partielle. Certes, nous ne trouvons pas chez Descartes d'analyse de la perception comparable à celles que nous donnera la Phénoménologie, montrant que la chose sensible se donne "par profils»; mais il dira plus tard que la sensation peut être claire, mais ne peut jamais être distincte. L'idée de voir entièrement n'a pas de signification s'agissant de la vision par les yeux du corps. Au contraire, l'intuition intellectuelle saisit ce qu'on pourrait appeler un atome logique: il s'agit d'une vérité indivisible, même si elle est constituée de plusieurs termes; elle ne comporte pas de parties, dont je connaîtrais les unes en ignorant les autres. C'est pourquoi, nous dit Descartes, un écolier qui a démontré une proposition mathématique en sait autant à son sujet que le plus grand mathématicien du monde.

S'il en est ainsi, rien n'est plus opposé que le discours démonstratif et la méditation, qui s'opposent comme la marche en avant, d'évidence en évidence, et le recreusement, la rumination, de ce qui a été entrevu, sans être entièrement vu. Une première série de justifications du style méditatif de l'itinéraire métaphysique peut facilement être trouvée; elles sont du reste étroitement liées. L'apprenti mathématicien n'a rien à détruire, alors que le métaphysicien doit lutter contre les préjugés, dont certains proviennent de la culture savante (comme la Scolastique), mais d'autres, bien plus nombreux et difficiles à surmonter, de l'expérience sensible et de l'état d'enfance, qui est et sera toujours notre condition première (on y reviendra). Il y a aussi ce qui relève du langage: celui-ci, né de et pour la vie commune, donc du règne des sens, risque à chaque instant de faire perdre au métaphysicien le bénéfice de ses découvertes. Nous en trouvons une illustration caractéristique après le Cogito. "Cette proposition: Je suis, j'existe, est nécessairement vraie, toutes les fois que je la prononce, ou que je la conçois en mon esprit». Aussitôt après, nous lisons: 
Mais je ne connais pas encore clairement ce que je suis, moi qui suis certain que je suis. De sorte que désormais il faut que je prenne soigneusement garde de ne pas prendre imprudemment quelque autre chose pour moi, et ainsi de ne point me méprendre dans cette connaissance, que je soutiens être plus certaine et plus évidente que toutes celles que j'ai eues auparavant ${ }^{23}$.

Alors que le mathématicien ne peut se tromper sur ce qu'il entend par «cercle» ou par «droite», puisqu'il en a posé les définitions, le métaphysicien risque à chaque instant d'être égaré par les mots: il est certain que Je suis; mais par «je » j'ai jusqu'ici entendu: cet individu que particulier, ou encore « un homme»; ce qui fait que, bien que l'affirmation «je suis» soit indubitable, je risque de m'égarer, de croire que j'ai découvert autre chose que ce que j'ai effectivement découvert. De là la nécessité, sans cesse affirmée par Descartes, de la lenteur, de la reprise, qui ne vient pas de ce que chaque étape est insuffisamment établie, mais de ce que, pour aller de l'avant, je dois confier à la mémoire et au langage ce qui a été objet d'une intuition.

Pourtant, ce n'est pas là l'unique justification de ce titre, Méditations, ni la plus intéressante. Il me semble possible de distinguer deux ordres dans les Méditations: l'ordre progressif de la démonstration et l'ordre régressif de la méditation, qu'on pourrait aussi nommer l'ordre de la philosophie première et l'ordre de la métaphysique. Les Méditations suivent la règle générale de l'ordre, inspirée de la démonstration mathématique, codifiée dans les Regulae et dans le Discours, et dont la définition la plus générale se lit dans les Secondes Réponses:

L'ordre consiste en cela seulement, que les choses qui sont proposées les premières doivent être connues sans l'aide des suivantes, et que les suivantes doivent être disposées de telle façon qu'elles soient démontrées par les seules choses qui les précèdent ${ }^{24}$.

Mais - bien que Descartes lui-même n'ait pas théorisé cet aspect de son discours, il faut le reconnaître - on peut discerner un autre mouvement, sans équivalent dans les sciences, qui consiste à mettre au jour ce qui était déjà là depuis le début, mais que nous n'apercevions pas. Pour le montrer, il faudrait reprendre tout le texte des Méditations, ce dont il ne saurait être question ici. Quelques rapides indications suffiront peut-être.

Lorsque la $\mathrm{II}^{\mathrm{e}}$ Méditation parvient au ego sum, c'est à coup sûr une vérité nouvelle, puisque, nous l'avons vu, la première journée avait entraîné un doute si profond que rien ne nous assurait qu'il serait possible d'y mettre fin: peut-être serons-nous conduits à reconnaître «qu'il n'y a rien 
au monde de certain». Et lorsque la nature de cet ego sera énoncée: «je ne suis précisément qu'une chose qui pense», cette vérité sera obtenue difficilement, contre la croyance spontanée, qui fait de moi « un homme», un être de chair et d'os qui se nourrit, marche et sent. Et cependant, cette chose dont toute l'essence est de penser, c'est bien celle qui était là dès la première ligne de la Première Méditation: car qu'est-ce que croire, avoir des opinions, douter, chercher, supposer, suspendre son jugement, sinon penser? Dès le début, Descartes (ou celui qui reprend sa démarche) était cet être dont l'existence est indubitable, et dont l'essence est de penser. Mais il n'y prenait pas garde.

De la même façon, considérons l'élévation vers Dieu qui clôt la III ${ }^{\mathrm{e}}$ Méditation, par laquelle j'éprouve à la fois la présence en moi de l'idée de Dieu et la distance infinie qui existe entre le créateur et sa créature. Elle vient au terme de la longue et difficile démonstration de l'existence de Dieu et, à l'évidence, ne pouvait venir avant. Pourtant, Dieu n'a pas été trouvé ailleurs qu'en moi, et l'idée de Dieu n'est pas différente de l'idée de moi-même:

Et certes on ne doit pas trouver étrange, que Dieu, en me créant, ait mis en moi cette idée pour être comme la marque de l'ouvrier empreinte sur son ouvrage; et il n'est pas aussi nécessaire que cette marque soit quelque chose de différent de ce même ouvrage. Mais, de cela seul que Dieu m’a créé, il est fort croyable qu'il m'a en quelque façon produit à son image et semblance, et que je conçois cette ressemblance (dans laquelle l'idée de Dieu se trouve contenue) par la même faculté par laquelle je me conçois moi-même, c'est-àdire que lorsque je fais réflexion sur moi [...] je connais que je suis une chose imparfaite $[\ldots]$ qui tend et aspire sans cesse à quelque chose de meilleur et de plus grand que je ne suis ${ }^{25}$.

Or, cette aspiration à une perfection que je ne possède pas, n'est-ce pas très précisément ce qui s'exprime au début des Méditations, comme déjà dans le Discours de la Méthode, à travers l'insatisfaction et l'incertitude? Mais, ici encore, ce qui était là dès le début est seulement à présent explicitement pensé. Il serait facile de montrer qu'il en va de même avec la volonté: s'il appartient à la IV Méditation de la penser et de montrer son rôle dans le jugement - ceci dans le cadre d'une question bien définie, celle de l'erreur - il suffit de relire la fin de la Première Méditation pour la trouver à l'œuvre: «je supposerai», «je penserai», «je me considérerai moi-même», «je demeurerai attaché à cette pensée», «il est en ma puissance de suspendre mon jugement ${ }^{26}$. Du reste, déjà dans le Discours, «l'histoire 
de [son] esprit», n'est-ce pas l'histoire de la libre volonté de Descartes qui nous a été narrée? Méditer, n'est-ce pas revenir sur les étapes passées, pour y mettre au jour ce qui y est déjà présent ${ }^{27}$ ?

La prise en compte de ce mouvement régressif qui met au jour ce qui était latent éclaire la question de la nature du «sujet» cartésien. Les commentateurs exclusivement attachés à la question du fondement de la science, surtout ceux qui ne veulent voir dans l'Ego cartésien que l'annonce, encore imparfaite, du Sujet transcendantal, ont insisté sur la «désindividualisation » de ce sujet. Le «Je» du «Je pense» n'est ni René Descartes, ni aucun homme individuel; il n'est même pas « un homme», cet habitant, parmi d'autres, de l'univers. Même, pour certains, il y aurait là une Pensée impersonnelle, dont les différents penseurs ne seraient que les manifestations ${ }^{28}$. Le mystérieux correspondant qui signe «Hyperaspistes» avait du reste suggéré une telle lecture du Cogito : "J'ajoute que vous ne savez pas si c'est vous-même qui pensez (ipse cogites), ou si c'est l'âme du monde qui est en vous qui pense, comme le voulaient les Platoniciens ${ }^{29}$.

Dans sa réponse, Descartes ne dit rien sur cette question. Non sans motif: la démarche même des Méditations exclut l'idée d'une pensée impersonnelle. Bien plus: elle ôte tout sens à la distinction du «sujet » universel et du « moi» individuel. Pour s'en persuader, il n'est que de tenter de répondre à la question: quand, à quel moment des Méditations, l'homme René Descartes laisserait-il la place au «sujet»? Il est impossible de le déterminer. Il y a bien

27. Que la progression soit moins une marche en avant que la mise en lumière de ce qui était déjà là, mais que le manque d'attention et les préjugés me masquaient, est explicitement affirmé à la fin de l'«analyse du morceau de cire»: «sa perception [...] n'est point une vision, ni un attouchement, ni une imagination, et ne l'a jamais été, quoiqu'il le semblât auparavant, mais seulement une inspection de l'esprit, qui peut être imparfaite et confuse, comme elle était auparavant, ou bien claire et distincte, comme elle est à présent, selon que mon attention se porte plus ou moins aux choses qui sont en elle, et dont elle est composée » (AT, VII, p. 31 et IX, p. 25).

28. «Cette pensée est-elle pour autant individualisée par elle-même ou traduit-elle seulement les manifestations empiriques d'un principe impersonnel et transcendant? Pour Schelling, par exemple, le Cogito réfléchit sur une pensée objective préexistante, et son authentique transcription serait: Cogitatur, Es denkt in mir. "Le véritable sujet du Je pense, déclare également L. Brunschvicg, c'est, pour un cartésianisme non systématiquement restreint, la pensée elle-même, capable de soutenir effectivement l'univers mathématique" » (G. Lewis, L'individualité selon Descartes, Paris, Vrin, 1950, p. 98). Plus près de nous, A. Renaut, dans L'ère de l'individu (Paris, Gallimard, 1989, p. 110), relève l'existence de «deux logiques possibles d'interprétation du moment cartésien: une logique d'interprétation impersonnelle», qu'il illustre lui aussi en citant le texte de Brunschvicg ( la pensée elle-même» comme "véritable sujet du je pense») et « une seconde logique mettant l'accent sur l'intériorité du cogito, au sens où un Maine de Biran pourra créditer Descartes d'avoir dans une certaine mesure procédé à partir du sens intime».

29. Lettre de Hyperaspistes, juillet 1641, AT, III, p. 403 "adde quod nescias si ipse cogites, an anima mundi, quae sit in te, cogitet, ut volunt Platonici» (nous traduisons). 
un moment où il se dépouille de ce qu'il croyait être lui : c'est le moment du doute, dans la Première Méditation; mais il ne se dépouille pas de son individualité. Comment le pourrait-il, puisque c'est lui-même qui doute. Il ne s'agit pas pour Descartes (et pour quiconque philosophe avec lui) de se dépouiller de lui-même, mais de découvrir sa propre essence.

Le titre des Principes de la Philosophie en énoncera clairement le dessein; c'est bien à leur propos qu'on peut parler de "philosophie première "; aussi le texte se limite-t-il, sauf rares exceptions, à ce qui est nécessaire à cette entreprise. Les Méditations, concurremment à cette tâche, se livrent à une autre: «la connaissance de Dieu et de soi-même», ou plutôt de la connaissance de soi-même en tant qu'elle est indissolublement liée à la connaissance de Dieu: se connaître, c'est se connaître comme un être fini qui a en lui l'idée de l'infini, qui ne découvre ce qu'il est qu'en mettant au jour son rapport à Dieu. Par ce côté, c'est bien d'une méditation qu'il s'agit. Et cette méditation est le fait de l'homme Descartes - même s'il appartient à chacun de refaire, à son tour et dans la solitude, le même parcours.

Ce n'est pas dire que nous retrouvons tous les aspects de la méditation religieuse. Celle-ci ne tend pas seulement à parvenir à la conscience de soi comme créature finie et dépendante, mais aussi comme créature pécheresse. Il va sans dire que cette dimension est totalement absente de la méditation cartésienne. Mais il est une autre différence, dont il faut prendre acte. Alors que la méditation du croyant est une tâche sans cesse reprise, il semble qu'il n'y ait ici rien de tel: du mouvement d'admiration et d'adoration qui constitue le point d'orgue de la III ${ }^{\mathrm{e}}$ Méditation, il n'y a plus aucun signe dans les écrits ultérieurs. S’il est vrai que nous ne pouvons connaître les pensées et pratiques intimes de Descartes, il affecte dans ses dernières années un certain mépris pour la métaphysique, et semble tourné vers la connaissance des choses et la vie en ce monde. Faut-il parler d'un tempérament peu porté vers l'introspection et la rumination? À moins que ce ne soit une conséquence de sa métaphysique elle-même? N'oublions pas que Descartes professe constamment à partir de 1630 l'incompréhensibilité de Dieu. Peut-être estime-t-il qu'on ne peut aller au-delà du terme qu'atteint la III $^{\mathrm{e}}$ Méditation? Dieu avait donné à Adam le jardin d'Eden pour le cultiver; son péché a été de vouloir partager la science divine. Peut-être le philosophe doit-il, lui aussi, cesser de méditer et se rendre «comme maître et possesseur de la nature». Ce projet "prométhéen » est peut-être humilité: «Je n'ai jamais traité de l'infini que pour me soumettre à lui » ${ }^{30}$.

30. Lettre à Mersenne du 28 janvier 1641, AT, III, p. 293. 
Quod vitae sectabor iter? [quel chemin suivrai-je en la vie?] ${ }^{31}$.

S'il est vrai que les Méditations ne répondent pas seulement à la question de la philosophie moderne «comment la science est-elle possible?», mais aussi à la plus ancienne injonction "connais-toi toi-même», les trois dernières parties de l'ouvrage peuvent décevoir. Certes, la IV apporte cet élément décisif qu'est l'affirmation de la libre volonté du "sujet». Mais la recherche reste strictement déterminée, et donc limitée, par la problématique: comment accorder le fait indéniable de l'erreur, fait sans lequel la recherche n'aurait pas été entreprise, avec la perfection divine? Quant à la $\mathrm{VI}^{\mathrm{e}}$ et dernière, elle démontre l'existence des corps, démonstration dans laquelle le rapport du sujet à son corps joue un rôle déterminant. Non seulement j'ai un corps, mais «la nature m'enseigne aussi par ces sentiments de douleur, de faim, de soif, etc., que je ne suis pas seulement logé dans mon corps, ainsi qu'un pilote en son navire, mais, outre cela, que je lui suis conjoint très étroitement, et tellement confondu et mêlé, que je compose comme un seul tout avec lui ${ }^{32}$.

Cette première et très remarquable "phénoménologie du corps propre» n'est cependant, elle aussi, développée qu'autant qu'il est requis pour la démonstration de l'existence du monde matériel, selon le principe qui fait prévaloir l'«ordre des raisons» sur l'«ordre des matières». C'est en vain que nous chercherions ici une réponse à la question: qu'est-ce pour moi, dont toute l'essence est d'être une chose qui pense, que d'avoir (et, en quelque manière, d'être) un corps? C'est à l'ultime ouvrage de Descartes, Les Passions de l'âme, qu'il appartient de répondre à cette interrogation.

L'article XVIII introduit le concept de volonté, en reprenant et en complétant ce qu'on lisait dans les Méditations:

Derechef, nos volontés sont de deux sortes. Car les unes sont des actions de l'âme, qui se terminent dans l'âme même, comme lorsque nous voulons aimer Dieu, ou généralement appliquer notre pensée à quelque objet qui n'est point matériel. Les autres sont des actions qui se terminent dans notre corps, comme de cela seul que nous avons la volonté de nous promener, il suit que nos jambes se remuent et que nous marchons ${ }^{33}$.

L'article XLI («Quel est le pouvoir de l'âme au regard du corps»), en un curieux mouvement, affirme d'abord la toute-puissance de la volonté:

31. Troisième songe de la nuit du 11 novembre 1619 relaté dans les Olympiques, AT, X, p. 183; le vers cité est du poète latin Ausone (Idylles, 15, 1); sur cette citation, voir Descartes, Étude du bon sens. La recherche de la vérité et autres écrits de jeunesse, V. Carraud, G. Olivo (éd.), Paris, PUF, 2013, p. 111, n. 14.

32. AT, VII, p. 81 et IX, p. 64.

33. AT, XI, p. 342-343. 
«Mais la volonté est tellement libre de sa nature, qu'elle ne peut jamais être contrainte", avant d'effectuer une marche arrière. Après avoir réaffirmé la puissance de la volonté par rapport à l'entendement: ses pensées «sont absolument en son pouvoir, et ne peuvent qu'indirectement être changées par le corps» (ce qui contient déjà une réserve), il est dit que nos passions «dépendent absolument des actions qui les produisent, et elles ne peuvent qu'indirectement être changées par l'âme $[\ldots] »^{34}$.

Pour comprendre quelle est la puissance - et l'impuissance - de la volonté, il faut rappeler la nature des passions, à la définition desquelles est consacré tout le début de l'ouvrage. Alors que la tradition les rapporte aux facultés inférieures de l'âme, Descartes les rapporte au corps. Certes, elles ont bien leur siège dans l'âme - ce sont des cogitationes, et à ce titre elles participent de l'indubitabilité du «Je suis» - mais leur cause est dans le corps. Par exemple, la vue d'une «figure fort étrange» (entendons : l'image dans le cerveau) provoque une série de mouvements qui aboutissent à la fuite. Chez l'animal, c'est là le tout du processus. Chez l'homme, ces différents mouvements se traduisent dans l'âme par l'émotion de la peur, ensuite par le désir de fuir. Quel est alors le pouvoir de la volonté? On a vu que, normalement, elle a le pouvoir de commander aux jambes de marcher ou de demeurer immobiles. Mais lorsque la passion est forte, ce pouvoir n'existe plus, comme chacun en a fait l'expérience: on ne cesse pas d'avoir peur sur commande. La volonté doit alors user de ruse en utilisant le pouvoir qu'elle a sur ces idées. Par exemple, comme l'expose l'article XLV, je dois me représenter «les raisons, les objets ou les exemples qui persuadent que le péril n'est pas grand», et surtout susciter en moi les passions contraires, en me représentant la honte qu'il y aurait à fuir, ou la gloire que me vaudrait d'avoir affronté le danger. Cette stratégie a pourtant ses limites, car la passion subvertit mon jugement, en me représentant l'objet plus dangereux (s'il s'agit de la peur) ou plus aimable (s'il s'agit de l'amour), et ainsi du reste, qu'il n'est effectivement (art. XLVI). C'est pourquoi, comme Descartes l'écrivait déjà en 1640, s'il est vrai qu'il n'y a rien qui soit en notre pouvoir que nos pensées, cela ne signifie pas qu'elles sont entièrement en notre pouvoir ${ }^{35}$.

Ce rappel permet de dégager une idée décisive : la volonté est toujours précédée par la passion. Elle n'intervient que pour approuver, réorienter, combattre des passions déjà là. Si, dans l'ordre de la recherche métaphysique, l'homme est découvert comme âme avant de se révéler âme

34. AT, XI, p. 359.

35. Lettre à Mersenne du 3 décembre 1640, AT, III, p. 249. 
étroitement unie à un corps, dans l'ordre de l'existence effective, l'homme est âme dominée par le corps avant de disposer de la puissance de penser et de juger. On sait l'importance du thème du préjugé chez Descartes. Par l'importance qu'il accorde à la lutte contre les préjugés, il est, de l'avis unanime, le précurseur de la «Philosophie des Lumières». Il faut cependant relever que, contrairement à la forme vulgarisée de cette pensée des Lumières, il ne croit pas que l'Humanité pourra dépasser l'ère des préjugés. Ce n'est pas sans raison qu'il se plaît à répéter que «nous avons été enfants avant que d'être hommes». Relisons l'article 1 des Principes de la Philosophie:

Comme nous avons été enfants avant que d'être hommes, et que nous avons jugé tantôt bien, tantôt mal des choses qui se sont présentées à nos sens lorsque nous n'avions pas encore l'usage entier de notre raison, plusieurs jugements ainsi précipités nous empêchent de parvenir à la connaissance de la vérité $[\ldots]^{36}$.

Le jugement est toujours second par rapport au pré-jugé. C'est là la condition humaine, de façon indépassable. Certes, l'âme est présente dès la conception. Mais elle est impuissante. Dès la vie fœetale, commence le processus de formation des passions (par exemple, une frayeur éprouvée par la mère peut provoquer chez l'enfant ce que nous appellerions une phobie). Et ce «conditionnement» va se poursuivre pendant l'enfance: on connaît l' «auto-analyse » de Descartes à propos de son penchant pour les femmes qui louchent. Lorsque l'homme parviendra à l'âge de raison, il aura déjà un lourd fardeau de préjugés et de passions, inscrit dans son corps, et par suite dans son âme.

Les Méditations nous faisaient découvrir l'homme comme esprit; les Passions nous parlent de l'homme, en tant qu'il est intimement uni à un corps. L'Homme en général, non Descartes en particulier - d'autant que sa méthode n'a certes rien d'introspectif. Il est pourtant intéressant de mettre en relation cette connaissance de l'homme avec le récit autobiographique du Discours de la Méthode : celui-ci illustre le discours sur l'homme, qui, en retour, l'éclaire.

"J'ai été nourri aux lettres dès mon enfance». Ainsi commence le récit dont Descartes espère "qu'il sera utile à quelques-uns, sans être nuisible à personne " ${ }^{37}$. "Mais", poursuit-il, «sitôt que j'eus achevé ce cours des études [...], je changeais entièrement d'opinion». Il est embarrassé de

36. AT, IX ${ }^{2}$, p. 25; latin, VIII, p. 5 .

37. Discours de la méthode, AT, VI, p. 4. 
doutes et d'erreurs. Descartes n'a choisi ni d'étudier au Collège de la Flèche (c'est son père qui l'y a envoyé), ni que tel soit le contenu des études. Pourtant cette situation va avoir un rôle déterminant, comme il le déclare explicitement. Après avoir parlé de ceux qui «se content[ent] de suivre les opinions des autres », plutôt que d' " en chercher eux-mêmes de meilleures", il ajoute:

Et pour moi, j'aurai sans doute du nombre de ces derniers, si je n'avais jamais eu qu'un seul maître, ou que je n'eusse point su les différences qui ont été de tout temps entre les opinions des plus doctes ${ }^{38}$.

Les insuffisances de l'enseignement reçu sont donc la felix culpa, sans laquelle la philosophie cartésienne n'aurait pas existé. Le Discours ne nous dit pas ce qu'il a lu dans le "grand livre du monde»; mais ses voyages dans différents pays, de diverses religions, en pleine guerre de Trente Ans, n'ont pu que renforcer sa déception et son sentiment d'incertitude. Quand la décision de chercher par lui-même est-elle intervenue? Est-ce quand il a décidé de quitter l'étude pour parcourir le monde? Quand a-t-il «pris la résolution d'étudier aussi en [lui]-même», comme il est dit à la fin de la première partie? Il est vraisemblable que, soit stylisation du récit, soit illusion rétrospective, ces décisions - comme toutes les décisions - ont été prises un peu à l'aveuglette, et que c'est après coup que Descartes s'attribue un projet constant de parvenir à la vérité par ses seules forces. Est-ce à dire que le libre exercice de la volonté est une illusion?

Peut-être n'a-t-on pas prêté une attention suffisante à un détail de la III ${ }^{\mathrm{e}}$ partie du Discours de la Méthode. Lorsque Descartes annonce la «morale par provision», il parle de "trois ou quatre maximes». Trois, ou quatre? Cette formulation s'explique par le fait qu'elles ne se situent pas exactement sur le même plan. Les trois premières, bien que Descartes les ait formées pour son propre usage, peuvent être adoptées par chacun de la même façon: obéir aux lois et coutumes du pays où l'on vit, être ferme et résolu en ses actions, se vaincre plutôt que la fortune, cela vaut pour tous. Il en va de même, en un sens, de la dernière maxime: faire une revue des différentes occupations des hommes en cette vie, pour tâcher de faire choix de la meilleure. Mais chacun n'aura peut-être pas la chance de Descartes:

et sans que je veuille rien dire de celle des autres, je pensai que je ne pouvais mieux que de continuer en celle-là même où je me trouvais, c'est-à-dire d'employer toute ma vie à cultiver ma raison, et à m'avancer, autant que je pourrais, en la connaissance de la vérité, suivant la méthode que je m’étais

38. Discours de la méthode, AT, VI, p. 16. 
prescrite. J'avais éprouvé de si extrêmes contentements, depuis que j'avais commencé à me servir de cette méthode, que je ne croyais pas qu'on pût en recevoir de plus doux, ni de plus innocents, en cette vie ${ }^{39}$.

Si l'usage de la raison est précédé par l'enfance, c'est-à-dire par le règne des sens et des préjugés, la décision de la volonté est toujours précédée par d'autres décisions, prises dans une demi-obscurité (il est manifeste que lorsque Descartes est parti parcourir le monde, il ne savait pas ce qu'il allait trouver ni, sans doute, ce qu'il cherchait). Mais ce que peut - et doit - faire la volonté éclairée, c'est faire retour sur l'itinéraire parcouru afin de le valider («je le ferais encor, si j'avais à le faire» dit, presque au même moment, le héros cornélien ${ }^{40}$ ) - ou à le renier. Mais dans le cas de Descartes, aucune conversion ne sera nécessaire.

Nous découvrons donc une parfaite concordance entre ce que nous apprennent Les Passions de l'âme au sujet de l'Homme, en tant qu'il est une âme étroitement unie à un corps, et la manière dont Descartes pense sa propre vie. Il apparaît en outre que l'ordre de la théorie et celui de la pratique sont bien différents. Le savoir doit commencer par la «table rase», par la mise en doute de toutes les connaissances; il a un commencement absolu. La vie, au contraire, est toujours déjà commencée. J'ai tel corps, telles expériences enfantines, telle éducation, telles passions. La volonté ne peut qu'assumer ou renier son passé, dénouer les liens qui ont été noués et en construire d'autres. Elle n'est ni première, ni toute puissante.

Peut-être cette rapide étude des rapports entre l'homme René Descartes et ce que sa doctrine nous apprend au sujet de l'âme humaine aura-t-elle contribué à corriger deux représentations de notre philosophe: la représentation commune, pour laquelle être cartésien, c'est ne considérer en l'homme que la raison, tenir les passions pour inessentielles et nuisibles à la vie de l'esprit ${ }^{41}$, et celle d'un certain courant philosophique qui estime que Descartes a doté (ou, pour être fidèle à la signification véritable du Cogito, aurait dû doter) le Sujet d'un pur pouvoir de connaître - voire d'un pouvoir constituant qui va à l'encontre du cartésianisme réel. Non, Descartes ne dit pas, ne peut pas dire, Es denkt in mir. C'est lui qui pense, et qui doit user au mieux de sa pensée non seulement dans la constitution du savoir, mais dans son existence. C'est là, me semble-t-il, la signification de cette «histoire de [son] esprit» que présente le premier ouvrage qu'il a publié.

39. Ibid., p. 27.

40. Le Cid, acte III, scène 4, vers 878 .

41. Voir à ce sujet l'intéressant petit ouvrage de D. Kambouchner: Descartes n'a pas dit, Paris, Les Belles Lettres, 2015, chap. 17: «La raison se passe d'émotions". 
Certes, nous n'avons là que l'histoire de son esprit: s'il donne un rôle important aux passions, il ne nous dit rien des siennes. Le Discours, ce n'est pas les Confessions, ni celles d'Augustin, ni celles de Rousseau. Ceci en partie parce que l'époque n'est pas propre au coming out, en partie parce que la passion la plus profonde et la plus constante est chez lui celle qu'il manifeste à travers toute son œuvre: la passion de la vérité.

Anne-Marie Boudot 